\title{
EDITORIAL
}

\section{Family Physicians, Nurse Practitioners, Physician Assistants, and Scope of Practice: Who Will Decide?}

\author{
Joshua Freeman, MD
}

(Fam Med. 2019;51(4):305-7.)

doi: 10.22454/FamMed.2019.356702

$\mathbf{T}$ wo issues with a long history of discussion in our discipline - the appropriate role of nurse practitioners (NPs) and physician assistants (PAs) in family practices, and the appropriate scope of a family physician's practice-are addressed by a study in this issue of Family Medicine by Drs Dai, Ingram, and Peterson. Their paper provides new information about the impact of nurse practitioners (NPs) and physician assistants (PAs) on both the number of patients a physician's practice cares for (panel size) and the scope of practice of family physicians. ${ }^{1}$

The stronger data is about the prevalence of NPs and PAs in family medicine practices. Because the study surveyed all FPs sitting for the ABFM recertification exam in 2013 through 2016 , it had a $100 \%$ response rate, and nearly $70 \%$ reported having NPs and PAs in their practices. Less than half of the responding physicians were able to estimate their panel size, but panel size was higher in those working with NPs and PAs who answered this question-an unsurprising finding. This paper does not include any information on the cost associated with having these additional providers, so it is not possible to assess the cost increase relative to the increase in the number of patients. Indeed, there is not consensus about the appropriate number of patients that should be cared for by a physician, NP, or PA. It is obviously related to scope of practice, since the more things you do for your patients, the fewer

patients you may be able to care for. Previous research has shown, however, that the numbers arbitrarily set by health system employers are probably higher than is consistent with high-quality care. ${ }^{2,3}$ It is also unclear how often patients in these practices were assigned directly to the advanced practice providers or whether these providers cared only for patients assigned to the physician.

The physicians surveyed also answered questions about their scope of practice. While $90 \%$ care for adults over 65 and teenagers, only $7 \%$ perform deliveries or provide emergency care. About one-third care for patients in the hospital (results presented in Appendix Table 2). Those physicians whose practices included NPs and PAs reported a wider scope of care, but measured on the 0 to 30 scale of the Scope of Practice for Primary Care (SP4PC), the increase was only 0.53 points. While statistically significant, the clinical significance is questionable. A 2015 study indicates that greater scope of practice may make a clinically significant difference by decreasing costs and hospitalizations, but the current study does not show a reduced rate of hospitalization. ${ }^{4}$ Probably the type of practice had a greater influence on scope of care than the presence of NPs and PAs, but the overall SP4PC range was small, from 13.5 to 16.8 .

The American Academy of Family Physicians (AAFP), the largest organization representing family doctors, has a clear policy 
against independent practice for $\mathrm{NPs},{ }^{5}$ although it supports NPs (and PAs) within physician practices. Relatively few family physicians are self-employed (under $20 \%)^{6}$ and large percentages are employed by health systems rather than physician groups. In some states, NPs (and sometimes PAs) have independent practice privileges, while in many others they require a sponsoring physician. They may practice in the same office as the physician, or in remote sites, including isolated rural ones. Also, a new degree, doctor of nursing practice (DNP), is now being awarded to most recent NP graduates. This is a clinical degree (like the MD, and unlike the research-focused $\mathrm{PhD}$ ) but means that these new NPs can be called doctors, which probably does not reduce patient confusion.

The actual evidence for the quality of care impact of independent practice for NPs is uncertain. Nursing journals demonstrate more positive outcomes, but most studies have looked at acute care rather than chronic disease management. ${ }^{7}$ The appropriate, or even typical, scope of care for either FPs or NPs (or PAs) is difficult to assess from studies of existing practices because they are so varied. The AAFP emphasizes that family physicians receive much more extensive clinical training, and thus have greater ability to care for more complex patient problems. This benefit is only realized, however, in settings in which the patients have complex problems. Anecdotal stories supporting any assertion or analysis abound, providing reinforcement for preconceived notions. What is clear is that family physicians, like other physicians whose practice overlaps with that of nonphysician providers, cannot have it both ways, arguing that their greater training makes them able to care for more complex patients and have a wider scope of practice (and a higher salary), but then restricting their practices to provide only those services that an NP can do as well.

Sometimes it may be the physician choosing a narrower practice, possibly for lifestyle reasons, but health system employers often restrict the scope of practice of FPs, with some doctors providing only outpatient care while others provide only inpatient care. Indeed, these hospitalists are now following the shiftwork model, with "nocturnists" and other new titles. It is also not clear if a provider with a more limited scope of practice is happier or has less burnout. Maybe not getting up in the middle of the night makes life easier, but providing only routine care far below your level of training can be very unsatisfying.

The best interests of the health system are clearly not always the same as the best interests of patients. This reminds us of the 1960s, when recognition of the limitations of specialist and subspecialist care led to the development of family medicine. A person is more than the sum of their parts, more than a collection of organs that can each be treated. The systems perspective elucidated by George Engel includes the pieces that make up people, but also the whole person and the larger contextdyad, family, community—in which they live. ${ }^{8,9}$ If each specialist only cares for their part of the person, who takes care of the whole person? If a provider takes care of a patient only part of the time, are we perhaps sacrificing quality of patient care on an imagined altar of efficiency?

This question is the key one for health care providers, for their employers, and most importantly for the people and communities they care for. The Dai, et al paper provides us with some new information about the high prevalence of NPs and PAs in family practices, and a bit about scope of practice, but it falls short of answering the most important questions about how primary care teams should work. If family physicians do have a special roleand I believe that they do-they must make that case. If not, others will define their role, not just advocates for NPs or PAs, but, more importantly, health systems and insurers. If our scope of practice as family physicians is changing and becoming more narrow, it is not because of NPs and PAs; it is because we either want it to be or we are unwilling to stand up to those who are narrowing it.

CORRESPONDENCE: Address correspondence to Dr Freeman at jfreeman@kumc.edu.

\section{References}

1. Dai M, Ingram R, Peterson L. Variations in scope of practice and patient panel size of family physicians who work with nurse practitioners or physician assistants. Fam Med. 2019;51(4):311-318.

2. Altschuler J, Margolius D, Bodenheimer T, Grumbach K Estimating a reasonable patient panel size for primary care physicians with team-based task delegation. Ann Fam Med. 2012;10(5):396-400. 
3. Raffoul M, Moore M, Kamerow D, Bazemore A. A Primary care panel size of 2500 is neither accurate nor reasonable. J Am Board Fam Med. 2016;29(4):496-499.

4. Bazemore A, Petterson S, Peterson LE, Phillips RL Jr. More comprehensive care among family physicians is associated with lower costs and fewer hospitalizations. Ann Fam Med. 2015;13(3):206-213.

5. American Academy of Family Physicians. Nurse Practitioners. https://www.aafp.org/about/policies/all/nursepractitioners.html. Accessed March 4, 2018.

6. Peckham C. Medscape Family Physician Compensation Report 2018 (Slide 7). https://www.medscape.com/ slideshow/2018-compensation-family-physician-6009655. Accessed March 4, 2019.
7. Greene J. Nurse practitioners provide quality primary care at a lower cost than physicians. Manag Care. 2018;27(11):34.

8. Engel GL. The need for a new medical model: a challenge for biomedicine. Science. 1977;196(4286):129-136.

9. Engel GL. The clinical application of the biopsychosocia model. Am J Psychiatry. 1980;137(5):535-544.

\section{Innovative Efforts Toward High Performing Academic Family Medicine Practices Call for Papers for a Special Issue}

Family Medicine requests submissions of original papers describing innovations that have improved one or more aspects of academic family medicine practice.

Submitted papers can address efforts to either optimize the patient and/or clinician experience within the practice or to improve the practice's outreach and community impact. Submissions may be original articles, brief reports, or narrative essays. Our goal for this special issue is to publish manuscripts with robust descriptions of the intervention and the criteria by which they have been evaluated. Narrative essays should relate stories about the experiences of faculty, staff, or students working in such settings. All papers must include objective evaluation data to support the value of the innovation, ideally over multiple years.

Submission Deadline: July 31, 2019.

All submissions should comply with the journal's instructions for authors: https://journals.stfm.org/familymedicine/authors/. While our standard word limits apply to these submissions, articles may include one or more appendices to allow more detailed descriptions of practice innovations. 